\title{
Actuator Fault Detection and Accommodation in Distributed Parameter Systems
}

\author{
Wenying $\mathrm{Mu}^{*}$ and Baotong Cui
}

Key Laboratory of Advanced Process Control for Light Industry (Ministry of Education), Jiangnan University, 1800 Lihu Road, Wuxi, 214122, China

\begin{abstract}
This paper presents a general framework for model-based fault detection and accommodation for a class of distributed parameter systems with control actuator faults. A set of dedicated observers, each with its own time-varying threshold is constructed to detect the occurrence of fault in the corresponding actuator and reduce fault detection time. Additionally, an adaptive diagnostic observer is then designed and analyzed to estimate each individual actuator fault. Based on the online approximation of fault parameter, automated control reconfiguration architecture has been developed to accommodate actuator fault and to preserve closed-loop stability. An example has been discussed using the proposed scheme to demonstrate effectiveness of the method.
\end{abstract}

Keywords: Actuator fault, Distributed parameter systems, Fault accommodation, Fault detection.

\section{INTRODUCTION}

Industrial processes have increased in complexity over the recent years due to the automation of the used control techniques, especially in the chemical and biological processes. In all of these processes, security and reliability of the system is critical. A fault could occur at any location or for any component used within the system. For example, a fault could occur with an actuator or sensor used in the system or in the defined system parameters. If not handled properly and in a timely manner, such a fault often leads to degradation in the processing operations. As a result, fault detection and accommodation within a reasonable timeframe is extremely important to ensure system safety.

Over the past few decades, a variety of methods have been developed and discussed in the literature extensively [1-6], which specifically detect and troubleshoot problems associated with actuators in some systems. Huang and $\mathrm{Yu}$ [2] presented an adaptive full-order observer to detect and estimate actuator fault for the Lur'e differential inclusion system. Hajiyev [3] considered a new approach based on generalized Rayleigh quotient for testing innovation covariance for Kalman filter applied to actuator and sensor fault detection. Du [4] proposed a method which exploits analytical redundancy in nonlinear systems through state observer design to isolate actuator and sensor faults. Yan and Edwards [5] studied an actuator fault detection and isolation scheme for a class of nonlinear systems with uncertainty based on a robust sliding mode observer.

Previous work on actuator fault detection and diagnosis has focused on lumped parameter systems described by ordinary differential equations (ODEs). However, distributed parameter systems (DPSs) are often used in many important industrial

*Address correspondence to this author at the Jiangnan University, 1800 Lihu Road, Wuxi, China; Tel: +86 15061873997; E-mail: weny815@126.com control processes characterized by spatial variations due to underlying physical phenomena $[7,8]$, such as convection, diffusion and phase dispersion [9]. These systems are typically modeled using partial differential equations. Control in DPSs often involves regulation of spatially distributed variables, such as temperature, pressure and concentration, using spatially distributed control actuators and measurement sensors [10]. However, it is worth noting that in particular actuator fault detection and accommodation in DPSs has received limited attention.

Over the recent years, particularly Demetriou [11, 12], Armaou [13], and EI-Frarra et al. [9, 10] studied actuator faulttolerant control in DPSs. Demetriou [11] considered an infinite dimensional adaptive detection observer, which was used to generate a residual signal to detect fault occurrence and assist with fault accommodation for DPSs. Armoaou [13] addressed a class of DPSs with component and actuator faults. An adaptive detection observer with a time-varying threshold and an adaptive diagnostic observer were proposed that provide fault declaration and estimation. Additionally, control reconfiguration scheme that accommodates component and actuator faults. However, the designed observers can only be used to detect total actuator faults and does not allow pinpointing the actuator where the fault occurred. This would result in all actuators being adjusted, including the healthy actuators in the system. Alternatively, EI-Frarra [12] also developed a methodology for design of an integrated, model-based fault diagnosis and reconfigurable control systems for PDEs with actuator faults and control constraints. A set of dedicated fault detection and isolation filters was constructed to replicate each of the fault-free behaviors at a given state. The residual of each filter is sensitive to fault for only a single actuator. However, each initial condition for the filter was required to be identical to the closed-loop system of the transformed reduced model in the absence of a fault. In such cases, knowledge of unmodeled dynamics and unknown initial conditions would be limited. 
Moreover, the use of fixed thresholds would increase the time required for fault detection.

Actuator fault detection and accommodation architecture are considered here for DPS, which can be decomposed into a finite dimensional slow subsystem and an infinite dimensional fast subsystem. A set of fault detection observers with appropriate time-varying thresholds are set up to monitor the slow subsystem for impending actuator faults. In order to avoid unnecessary adjustments of healthy actuators, residual signal of each observer is only sensitive to detect fault in the corresponding actuator even if the initial conditions were different. Dynamic (time-varying) threshold significantly reduces detection time. After the fault occurs, a set of diagnostic observers with adaptive schemes for the adjustable parameter are designed to capture dedicated actuator faults. Furthermore, actuator faults are accommodated with the use of control reconfiguration with an online estimate of the fault parameter. Therefore, the stability can be maintained for the closed-loop subsystem.

\section{PROBLEM FORMULATION}

The class of DPS under consideration here is described by the following equation:

$$
\begin{aligned}
\frac{\partial z(t, x)}{\partial t}= & \frac{\partial^{2} z}{\partial x^{2}}(t, x)+\sum_{i=1}^{n} b_{i}(x)\left[u_{i}(t)\right. \\
& \left.+H\left(t-T_{a_{i}}\right) f_{a_{i}}(t)\right]+\omega(z(t, x))
\end{aligned}
$$

subject to the boundary condition and initial condition

$$
\frac{\partial z}{\partial x}(t, 0)=\frac{\partial z}{\partial x}(t, h)=0, z(0, x)=z_{0}(x)
$$

The system is defined in the Hilbert space $X$, where $z(t, x)$ is the state; $x$ and $t$ are the spatial and time coordinate, respectively; $b_{i}(x)$ is the spatial distribution of the $i$ th actuator; $u_{i}(t)$ is the associated control signal; $\omega(z(t, x))$ is the nonlinear dynamics; $f_{a_{i}}(t)$ is a fault of $i$ th actuator, $i=1, \cdots n ; T_{a_{i}}$ is the time occurrence of the $i$ th actuator fault; $H\left(t-T_{a_{i}}\right)$ is the time profile of the $i$ th actuator fault, where

$$
H\left(t-T_{a_{i}}\right)=\left\{\begin{array}{l}
0, \text { if } t<T_{a_{i}}, \\
1, \text { if } t \geq T_{a_{i}} .
\end{array}\right.
$$

For convenience of analysis, let us rewrite the system (1) in the following form:

$$
\begin{gathered}
\dot{z}(t)=A z(t)+B\left[u(t)+H\left(t-T_{a}\right) f_{a}(t)\right]+\omega(z(t)), \\
z_{0} \in X,
\end{gathered}
$$

where $z(t)=z(t, x)$, operator $A$ is defined as $A \phi=\frac{d^{2} \phi}{d x^{2}}$, where $\phi, \frac{d \phi}{d x}$ are absolutely continuous on $(0, h)$ with domain

$$
D(A)=\left\{\phi \in L_{2}(0, h) \mid \frac{d^{2} \phi}{d x^{2}} \in L_{2}(0, h)\right. \text { and }
$$

$$
\left.\frac{d \phi}{d x}(0)=\frac{d \phi}{d x}(h)=0\right\} \text {. }
$$

$B$ is the control input operator. We set and $H\left(t-T_{a}\right) f_{a}(t)$

$$
=\left[H\left(t-T_{a_{i}}\right) f_{a_{i}}(t), \cdots, H\left(t-T_{a_{n}}\right) f_{a_{n}}(t)\right]^{T}, \omega(z(t)) \text { local- }
$$

ly satisfies Lipschitz condition $\omega(0)=0$.

It is assumed that the infinite dimensional state $z$ can be decomposed as [14]

$$
z=z_{s}+z_{f}=P_{s} z+P_{f} z
$$

where $z_{s}$ denotes the state of the finite-dimensional slow/unstable subsystem and $z_{f}$ denotes the state of the infinite dimensional fast/stable subsystem. $P_{s}$ and $P_{f}$ are projection operations yielding the following form:

$$
\begin{aligned}
& \frac{d z_{s}}{d t}=A_{s} z_{s}+B_{s}\left[u(t)+H\left(t-T_{a}\right) f_{a}(t)\right]+\omega_{s}\left(z_{s}+z_{f}\right), \\
& \frac{d z_{f}}{d t}=A_{f} z_{f}+B_{f}\left[u(t)+H\left(t-T_{a}\right) f_{a}(t)\right]+\omega_{f}\left(z_{s}+z_{f}\right), \\
& z_{s}(0)=P_{s} z(0), z_{f}(0)=P_{f} z(0),
\end{aligned}
$$

where $A_{s}=P_{s} A, B_{s}=P_{s} B, \omega_{s}=P_{s} \omega, A_{f}=P_{f} A, B_{f}=P_{f} B$, $\omega_{f}=P_{f} \omega . A_{s}=\operatorname{diag}\left\{\mu_{\mathrm{i}}\right\}$ is an $m$-dimensional diagonal matrix, $\left\{\mu_{1}, \cdots, \mu_{m}\right\}$ are the slow eigenvalues. The unbound differential operator $A_{f}$ is exponentially stable. By neglecting the fast/stable infinite dimensional subsystem $z_{f}$ in Eq. (4), we obtain the $m$ - dimensional slow system

$\frac{d z_{s}}{d t}=A_{s} z_{s}+B_{s}\left[u(t)+H\left(t-T_{a}\right) f_{a}(t)\right]+\omega_{s}\left(z_{s}\right)$.

Based on the above decomposition and model-reduction, first a fault detection filter needs to be designed that indicates the time of when the actuator fault occurs and allows to diagnosis related to the nature of the actuator fault. The secondary objective is to provide fault accommodation based on the estimate of the actuator fault.

\section{DETECTION OBSERVER}

To differentiate faults among different actuators, a dedicated fault detection and isolation filter can be designed for each mode. This allows the residual signal to be sensitive only to the actuator where the fault occurs.

In this case, the existence of a bounded, invertible matrix $Q_{s}$ is assumed, such that $Q_{s} B_{s}=T_{s}$, where $T_{s}$ is an identity matrix. Consider the transformation $n_{s}=Q_{s} z_{s}$, which transforms the slow system (5) as follows: 


$$
\begin{aligned}
\dot{n}_{s}(t)= & A_{s} n_{s}(t)+T_{s}\left[u(t)+H\left(t-T_{a}\right) f_{a}(t)\right] \\
& +v_{s}\left(Q_{s}^{-1} n_{s}(t)\right)
\end{aligned}
$$

where $v_{s}=Q_{s} \omega_{s}$.

In order to address the specific structure of the system, we can further decompose the slow state $n_{s}(t)$ as

$n_{s}(t)=n_{s_{1}}(t)+n_{s_{2}}(t)+\cdots n_{s_{m}}(t)$,

where $n_{s_{i}}=K_{s_{i}} n_{s}(t)$ as a one-dimensional state denotes the evolution of the $i$ th transformed slow mode. The orthogonal projection matrix $K_{s_{i}}$ projects $n_{s}(t)$ onto $n_{s_{i}}(t)$. In the above decomposition, for $i=1, \cdots, m$, the $i$ th transformed slow mode can be rewritten as follows:

$$
\begin{aligned}
\dot{n}_{s_{i}}(t)= & A_{s} n_{s_{i}}(t)+K_{s_{i}} T_{s}\left[u(t)+H\left(t-T_{a}\right) f_{a}(t)\right] \\
& +K_{s_{i}} v_{s}\left(\left(Q_{s} K_{s_{i}}\right)^{-1} n_{s_{i}}(t)\right) \\
= & A_{s} n_{s_{i}}(t)+K_{s_{i}} Q_{s} P_{s} B\left[u(t)+H\left(t-T_{a}\right) f_{a}(t)\right] \\
& +v_{s_{i}}\left(\left(Q_{s} K_{s_{i}}\right)^{-1} n_{s_{i}}(t)\right) \\
= & A_{s} n_{s_{i}}(t)+K_{s_{i}} Q_{s} P_{s} \sum_{i=1}^{n} b_{i}(x)\left[u_{i}(t)\right. \\
& \left.+H\left(t-T_{a_{i}}\right) f_{a_{i}}(t)\right]+v_{s_{i}}\left(\left(Q_{s} K_{s_{i}}\right)^{-1} n_{s_{i}}(t)\right)
\end{aligned}
$$

where $v_{s_{i}}=K_{s_{i}} v_{s}$. Since the operator $Q_{s} B_{s}$ has a diagonal structure, then we have $K_{s_{i}} Q_{s} P_{s} b_{j}(x)=0$ for $j \neq i$. It results in

$$
\begin{aligned}
\dot{n}_{s_{i}}(t)= & A_{s} n_{s_{i}}(t)+K_{s_{i}} Q_{s} P_{s} b_{i}(x)\left[u_{i}(t)+H\left(t-T_{a_{i}}\right) f_{a_{i}}(t)\right] \\
& +v_{s_{i}}\left(\left(Q_{s} K_{s_{i}}\right)^{-1} n_{s_{i}}(t)\right) .
\end{aligned}
$$

Throughout this paper, the following assumptions are given:

Assumption 1. The number of slow modes is equal to the number of actuators, i.e., $m=n$.

Assumption 2. Consider the above fault-free approximate slow system, then the proposed control signal is given by

$u_{i}(t)=-\left(K_{s_{i}} Q_{s} P_{s} b_{i}(x)\right)^{-1}\left[v_{s_{i}}\left(\left(Q_{s} K_{s_{i}}\right)^{-1} n_{s_{i}}(t)\right)+k_{i} n_{s_{i}}(t)\right]$,

where $k_{i}$ is a suitably chosen feedback gain such that the faultfree system (9) has satisfactory behavior.

Actuator fault detection can be accomplished by constructing an observer, which is combined with the approximate slow system (9) to produce a residual signal for detecting the fault. To this end, the dedicated detection observer takes the following form:

$\dot{m}_{i}(t)=A_{s} m_{i}(t)+K_{s_{i}} Q_{s} P_{s} b_{i}(x) u_{i}(t)-L_{i}\left(m_{i}(t)-n_{s_{i}}(t)\right)$, where $L_{i}$ is an appropriately chosen observer gain. The state observation error norm $\left\|e_{i}(t)\right\|=\left\|n_{S_{i}}(t)-m_{i}(t)\right\|$ serves as the residual signal.

Theorem 1. Consider an approximate, finite-dimensional closed-loop system (9), for which both Assumptions 1 and 2 hold. Then, the on-line observer given in (11) has the following properties.

(1) When all signals are nonzero, a fault is declared if the residual signal $\left\|e_{i}(t)\right\|$ exceeds the time-varying threshold $r_{i}(t)$ given by

$r_{i}(t)=e^{-\alpha_{i} t} \xi_{i}+\frac{1-e^{-\alpha_{i} t}}{\alpha_{i}} v_{i}$.

The actuator fault is declared at the detection time

$t_{a_{i}}=\inf _{t \geq T_{a_{i}}}\left\{\left\|e_{i}(t)\right\|>r_{i}(t)\right\}$

for $i=1, \cdots, m$.

(2) When $v_{s_{i}}\left(\left(Q_{s} K_{S_{i}}\right)^{-1} n_{s_{i}}(t)\right)=0$ and $n_{s_{i}}(0)=m_{i}(0)$, the residual signal $r_{i}(t)=0$ for all $0<t<T_{a_{i}}$, where $i=1, \cdots, m$. Then, $\left\|e_{i}(t)\right\|>0$, if and only if $f_{a_{i}}(t) \neq 0$.

Proof. (1) The state estimation error prior to fault occurrence $\left(t<T_{a_{i}}\right)$ can be expressed as:

$\dot{e}(t)=\left(A_{s}-L_{i}\right) e_{i}(t)+v_{s_{i}}\left(\left(Q_{s} K_{s_{i}}\right)^{-1} n_{s_{i}}(t)\right)$.

The solution of Eq. (14) is given as:

$e_{i}(t)=e^{\left(A_{s}-L_{i}\right) t} e_{i}(0)+\int_{0}^{t} e^{\left(A_{s}-L_{i}\right)(t-\tau)} v_{S_{i}}\left(\left(Q_{s} K_{S_{i}}\right)^{-1} n_{S_{i}}(\tau)\right) d \tau$.

The residual signal can be expressed as:

$$
\begin{aligned}
\left\|e_{i}(t)\right\| \leq & \left\|e^{\left(A_{s}-L_{i}\right) t} e_{i}(0)\right\| \\
& +\left\|\int_{0}^{t} e^{\left(A_{s}-L_{i}\right)(t-\tau)} v_{S_{i}}\left(\left(Q_{s} K_{S_{i}}\right)^{-1} n_{s_{i}}(\tau)\right) d \tau\right\| \\
& \leq e^{-\partial_{i} t}\left\|e_{i}(0)\right\|+\int_{0}^{t} e^{-\partial_{i}(t-\tau)}\left\|\nu_{s_{i}}\left(\left(Q_{s} K_{s_{i}}\right)^{-1} n_{s_{i}}(\tau)\right)\right\| d \tau \\
& \leq e^{-\alpha_{i} t} \xi_{i}+\frac{1-e^{-\alpha_{i} t}}{\alpha_{i}} v_{i},
\end{aligned}
$$

where $\partial_{i}$ is the spectrum bound for $\left(A_{S}-L_{i}\right), \xi_{i}$ is the upper bound in $\left\|e_{i}(0)\right\| \leq \xi_{i}$. The nonlinear dynamics $\left\|v_{s_{i}}\left(\left(Q_{s} K_{s_{i}}\right)^{-1} n_{s_{i}}(\tau)\right)\right\| \leq v_{i}$ for $i=1, \cdots, m$. Thus, the timevarying threshold is given by

$r_{i}(t)=e^{-\alpha_{i} t} \xi_{i}+\frac{1-e^{-\alpha_{i} t}}{\alpha_{i}} v_{i}$. 
An actuator fault is declared when the residual $\left\|e_{i}(t)\right\|$ exceeds the time-varying threshold $r_{i}(t)$. The detection time is given by

$t_{a_{i}}=\inf _{t \geq T_{a_{i}}}\left\{\left\|e_{i}(t)\right\|>r_{i}(t)\right\}$.

(2) When the initial state $n_{s_{i}}(0)=m_{i}(0)$, and $v_{s_{i}}\left(\left(Q_{s} K_{s_{i}}\right)^{-1} n_{s_{i}}(t)\right)=0$, one has $e_{i}(0)=0$ and $r_{i}(0)=0$.

Substituting this relation into Eq. 15 yields $e_{i}(0)=0$ for $0<t<T_{a_{i}}$. It follows that $r_{i}(t)=0$. Thereafter, for $t \geq T_{a_{i}}$, $\left\|e_{i}(t)\right\|>0$, if and only if $f_{a_{i}}(t) \neq 0$ from Eq. 17, thus indicating presence of an actuator fault in the system.

Remark 1. The transformed slow-subsystem of Eq. (9) differs from the one derived by [10]. In Eq. (9), a fault in actuator $j$ cannot influence the $i$ th slow mode, that is, even though there is a fault in actuator $i$, the mode $j(j \neq i)$ is still normal. Thus, the residual signal of $i$ th filter is sensitive to the $i$ th actuator fault, rather than all the actuators.

\section{ADAPTIVE DIAGNOSTIC SCHEME}

In the previous sections, the detection of actuator faults has been demonstrated. In this section, the next step towards the development of a fault diagnostic scheme is discussed. From an adaptive theory viewpoint, online approximations are used to adjust the system parameters when an actuator fault occurs. The objective is to establish a parameter adaptive law for $\hat{\theta}_{i}(t)$ so that online estimation of $\hat{f}_{a_{i}}(t)=\hat{\theta}_{i}(t) g_{i}(t)$ approximates the function $H\left(t-T_{a_{i}}\right) f_{a_{i}}(t)=H\left(t-T_{a_{i}}\right) \theta_{i} g_{i}(t)$. The convergence property for the adaptive diagnostic observer is formalized in Theorem 2 below.

Theorem 2. Consider the system described by (9) where the actuator fault occurs. Assume that there exists a diagnostic observer gain $\hat{L}_{i}$ that satisfies the Lyapunov equation:

$$
\left(A_{s}-\hat{L}_{i}\right)^{T} \Pi_{i}+\Pi_{i}\left(A_{s}-\hat{L}_{i}\right)=-Q_{i}
$$

with $\Pi_{i}=\Pi_{i}{ }^{T}>0$, and $\lambda_{\min }\left(Q_{i}\right)>0$ for $i=1, \cdots, m$. Then, the proposed adaptive diagnostic observer is given by

$$
\begin{aligned}
& \dot{\hat{n}}_{i}(t)=A_{s} \hat{n}_{i}(t)+K_{s_{i}} Q_{s} P_{s} b_{i}(x) u_{i}(t)-\hat{L}_{i}\left(\hat{n}_{i}(t)-n_{s_{i}}(t)\right) \\
& +K_{s_{i}} Q_{s} P_{s} b_{i}(x) \hat{\theta}_{i}(t) g_{i}(t)+v_{s_{i}}\left(\left(Q_{s} K_{s_{i}}\right)^{-1} n_{s_{i}}(t)\right), \\
& \hat{n}_{i}\left(t_{a_{i}}\right)=\hat{n}_{i_{0}}, \\
& \hat{\theta}_{i}(t)= \begin{cases}0, & t<t_{a_{i}}, \\
K_{s_{i}} Q_{s} P_{s} b_{i}(x) g_{i}(t) \Pi_{i} e_{d_{i}}(t), & t \geq t_{a_{i}},\end{cases} \\
& \hat{\theta}_{i}\left(t_{a_{i}}\right)=\hat{\theta}_{a_{i}}
\end{aligned}
$$

guarantee that the state diagnostic errors converge to zero, and all signals are bounded.
Proof. Consider $e_{d_{i}}(t)=n_{s_{i}}(t)-\hat{n}_{i}(t)$ is the state diagnostic error, the following is obtained:

$$
\begin{aligned}
\dot{e}_{d_{i}}(t)= & \left(A_{s}-\hat{L}_{i}\right) e_{d_{i}}(t)+K_{s_{i}} Q_{s} P_{s} b_{i}(x) H\left(t-T_{a_{i}}\right) f_{a_{i}}(t) \\
& -K_{s_{i}} Q_{s} P_{s} b_{i}(x) \hat{\theta}_{i}(t) g_{i}(t) \\
= & \left(A_{s}-\hat{L}_{i}\right) e_{d_{i}}(t)-K_{s_{i}} Q_{s} P_{s} b_{i}(x)\left(I-H\left(t-T_{a_{i}}\right)\right) \theta_{i} g_{i}(t) \\
& -K_{s_{i}} Q_{s} P_{s} b_{i}(x)\left(\hat{\theta}_{i}(t)-\theta_{i}\right) g_{i}(t) \\
= & \left(A_{s}-\hat{L}_{i}\right) e_{d_{i}}(t)-K_{s_{i}} Q_{s} P_{s} b_{i}(x) \varphi_{i}(t) \theta_{i} g_{i}(t) \\
& -K_{s_{i}} Q_{s} P_{s} b_{i}(x) \widetilde{\theta}_{i}(t) g_{i}(t),
\end{aligned}
$$

where $\varphi_{i}(t)=I-H\left(t-T_{a_{i}}\right)$ and $\widetilde{\theta}_{i}(t)=\hat{\theta}_{i}(t)-\theta_{i}$. The associated Lyapunov function is given as:

$$
V_{i}(t)=e_{d_{i}}^{T}(t) \Pi_{i} e_{d_{i}}(t)+\widetilde{\theta}_{i}(t) \Gamma_{i}^{-1} \widetilde{\theta}_{i}(t)+\lambda_{i} \varphi_{i}(t) P_{i}^{-1} \varphi_{i}(t),
$$

Where $\Gamma_{i}>0$ is the adaptive gain, $\lambda_{i}>0$ is a constant scalar. For $t>T_{a_{i}}, P_{i}$ relates the rate of change of $\varphi_{i}(t)$ and satisfies $\dot{\varphi}_{i}(t)=-P_{i} \varphi_{i}(t), \varphi_{i}\left(T_{a_{i}}\right)=I$. Using the fact that $\dot{\widetilde{\theta}}_{i}(t)=\hat{\theta}_{i}(t), i=1, \cdots, m$, along with (19) we have

$$
\begin{aligned}
\dot{V}_{i}(t)= & e_{d_{i}}^{T}(t)\left[\left(A_{s}-\hat{L}_{i}\right)^{T} \Pi_{i}+\Pi_{i}\left(A_{s}-\hat{L}_{i}\right)\right] e_{d_{i}}(t) \\
& -2 K_{s_{i}} Q_{s} P_{s} b_{i}(x) \varphi_{i}(t) \theta_{i} g_{i}(t) \Pi_{i} e_{d_{i}}(t) \\
& -2 K_{s_{i}} Q_{s} P_{s} b_{i}(x) \widetilde{\theta}_{i}(t) g_{i}(t) \Pi_{i} e_{d_{i}}(t) \\
& +2 \widetilde{\theta}_{i}(t) \Gamma_{i}^{-1} \dot{\tilde{\theta}}_{i}(t)-\lambda_{i} \varphi_{i}^{2}(t) \\
= & -e_{d_{i}}^{T}(t) Q_{i} e_{d_{i}}(t)-2 K_{s_{i}} Q_{s} P_{s} b_{i}(x) \varphi_{i}(t) \theta_{i} g_{i}(t) \Pi_{i} e_{d_{i}}(t) \\
& -\lambda_{i} \varphi_{i}^{2}(t) \\
\leq & -\lambda_{\min }\left(Q_{i}\right)\left\|e_{d_{i}}(t)\right\|^{2}-2 K_{s_{i}} Q_{s} P_{s} b_{i}(x) \varphi_{i}(t) \theta_{i} g_{i}(t) \Pi_{i} e_{d_{i}}(t) \\
& -\lambda_{i} \varphi_{i}^{2}(t) .
\end{aligned}
$$

By integrating from $t=t_{0}=0$ to $t=t_{1}<t_{a_{i}}$, we obtain

$$
\begin{aligned}
& \left\|\Pi_{i}^{\frac{1}{2}} e_{d_{i}}\left(t_{1}\right)\right\|^{2}+\left(\Gamma_{i}^{-1}\right)\left\|\widetilde{\theta}_{i}\left(t_{1}\right)\right\|^{2}+\int_{0}^{t_{1}} \lambda_{\min }\left(Q_{i}\right)\left\|e_{d_{i}}(\tau)\right\|^{2} d \tau \\
& +\int_{0}^{t_{1}} \lambda_{i} \varphi_{i}^{2}(\tau) d \tau \\
& \quad \text { For } t \in\left[t_{a_{i}}, \infty\right), \text { we also have } \\
& \quad\left\|\prod_{i}^{\frac{1}{2}} e_{d_{i}}(t)\right\|^{2}+\left(\Gamma_{i}^{-1}\right)\left\|\widetilde{\theta}_{i}(t)\right\|^{2}+\int_{t_{a_{i}}}^{t} \lambda_{\min }\left(Q_{i}\right)\left\|e_{d_{i}}(\tau)\right\|^{2} d \tau \\
& \quad \| \int_{t_{a_{i}}}^{t} 2 K_{s_{i}} Q_{s} P_{s} b_{i}(x) \varphi_{i}(t) \theta_{i} g_{i}(t) \Pi_{i} e_{d_{i}}(t) d \tau+\int_{t_{a_{i}}}^{t} \lambda_{i} \varphi_{i}^{2}(\tau) d \tau \\
& \leq\left\|\Pi_{i}^{\frac{1}{2}} e_{d_{i}}\left(t_{a_{i}}\right)\right\|^{2}+\left(\Gamma_{i}^{-1}\right)\left\|\tilde{\theta}_{i}\left(t_{a_{i}}\right)\right\|^{2} .
\end{aligned}
$$

Based on the above analysis, the bounds of the state error $e_{d_{i}}(t)$ and parameter estimator $\widetilde{\theta}_{i}(t)$ are readily obtained.

There exists a finite constant $c_{i}$ such that $\sup _{t \geq T_{a_{i}}} f_{a_{i}}(t)=$ 


$$
\begin{gathered}
\sup _{t \geq T_{a_{i}}} \theta_{i} g_{i}(t)=c_{i} \text {. Therefore, (21) becomes } \\
\dot{V}_{i}(t) \leq-\left(\lambda_{\min }\left(Q_{i}\right)\left\|e_{d_{i}}(t)\right\|^{2}+\lambda_{i}\left\|\varphi_{i}(t)\right\|^{2}\right) \\
+2 c_{i}\left\|K_{s_{i}} Q_{s} P_{s} b_{i}(x) \Pi_{i}\right\|\left\|\varphi_{i}(t)\right\|\left\|e_{d_{i}}(t)\right\| .
\end{gathered}
$$

Using the inequality $2 a b \leq \mu a^{2}+b^{2} / \mu$ for some $\mu>0$. By setting $\lambda_{i}=\left(c_{i}\left\|K_{s_{i}} Q_{s} P_{s} \Pi_{i}\right\|\right)^{2} / \lambda_{\min }\left(Q_{i}\right)$, it can be concluded that $\dot{V}_{i}(t) \leq 0$, by utilizing the Barbalat's lemma [15], we acquire $\lim e_{i}(t)=0$. Therefore, the state diagnostic errors asymptotic converge to zero. In addition, when $t \rightarrow \infty$, the parameter approximation $\hat{\theta}_{i}(t)$ asymptotically converges to $\theta_{i}$ for $i=1, \cdots, m$.

\section{FAULT ACCOMMODATION SCHEME}

So far the methods for detecting and diagnosing the actuator fault have been discussed. Next, in this section, a control reconfiguration to accommodate the fault is proposed. It is desirable to adjust the controller to preserve closed-loop system stability in the presence of the actuator fault. Due to the fact that the actuator fault is unknown, an estimation $\hat{\theta}_{i}(t)$ may be used instead of the fault parameter.

Theorem 3. Consider system (9) in which the fault actuator is declared, then the corresponding fault accommodating controller takes the form

$$
\begin{aligned}
u_{a c c_{i}}(t)=- & \left(K_{s_{i}} Q_{s} P_{s} b_{i}(x)\right)^{-1}\left[v_{s_{i}}\left(\left(Q_{s} K_{s_{i}}\right)^{-1} n_{s_{i}}(t)\right)\right. \\
& \left.+k_{i} n_{s_{i}}(t)\right]-\hat{\theta}_{i}(t) g_{i}(t)
\end{aligned}
$$

ensures the closed-loop system (9) satisfies the stability criteria and the residual signal is below its time-varying threshold.

Proof. In view of the control signal (10) for the closed-loop fault-free system

$\dot{n}_{s_{i}}(t)=\left(A_{s}-k_{i}\right) n_{s_{i}}(t)$.

When the fault is detected ( $\left.t \geq t_{a_{i}}\right)$, the ideal system can take the actuator fault into account as:

$$
\begin{aligned}
\dot{n}_{s_{i}}= & A_{s} n_{s_{i}}(t)+K_{s_{i}} Q_{s} P_{s} b_{i}(x)\left[u_{a c c_{i}}(t)+f_{a_{i}}(t)\right] \\
& +v_{s_{i}}\left(\left(Q_{s} K_{s_{i}}\right)^{-1} n_{s_{i}}(t)\right) \\
= & A_{s} n_{s_{i}}(t)+K_{s_{i}} Q_{s} P_{s} b_{i}(x)\left[u_{a c c_{i}}(t)+\theta_{i} g_{i}(t)\right] \\
& +v_{s_{i}}\left(\left(Q_{s} K_{s_{i}}\right)^{-1} n_{s_{i}}(t)\right) .
\end{aligned}
$$

By taking into account the fault accommodating controller (23), the closed-loop system is represented as follows:

$$
\begin{aligned}
\dot{n}_{s_{i}} & =\left(A_{s}-k_{i}\right) n_{s_{i}}(t)-K_{s_{i}} Q_{s} P_{s} b_{i}(x)\left(\hat{\theta}_{i}(t)-\theta_{i}\right) g_{i}(t) \\
& =\left(A_{s}-k_{i}\right) n_{s_{i}}(t)-K_{s_{i}} Q_{s} P_{s} b_{i}(x) \tilde{\theta}_{i}(t) g_{i}(t)
\end{aligned}
$$

If there exist positive definite matrix $P_{i}$ satisfying the Lyapunov equation

$$
\left(A_{s}-k_{i}\right)^{T} \Pi_{i}+\Pi_{i}\left(A_{s}-k_{i}\right)=-P_{i} .
$$

Consider the Lyapunov function candidate

$$
V_{a_{i}}(t)=n_{s_{i}}^{T}(t) \Pi_{i} n_{s_{i}}(t)
$$

whose time derivative is given by

$$
\begin{aligned}
\dot{V}_{a_{i}}(t)= & n_{s_{i}}^{T}(t)\left[\left(A_{s}-k_{i}\right)^{T} \Pi_{i}+\Pi_{i}\left(A_{s}-k_{i}\right)\right] n_{s_{i}}(t) \\
& -2 K_{s_{i}} Q_{s} P_{s} b_{i}(x) \tilde{\theta}_{i}(t) g_{i}(t) \Pi_{i} n_{s_{i}}(t) \\
\leq & -\lambda_{\min }\left(P_{i}\right)\left\|n_{s_{i}}(t)\right\|^{2} \\
& +2\left\|K_{s_{i}} Q_{s} P_{s} b_{i}(x) \Pi_{i}\right\|\left\|\widetilde{\theta}_{i}(t) g_{i}(t)\right\|\left\|n_{s_{i}}(t)\right\| .
\end{aligned}
$$

Since $\tilde{\theta}_{i}(t) g_{i}(t)=\hat{f}_{a_{i}}(t)-f_{a_{i}}(t)$, we assume that $\sup _{t \geq T_{a_{i}}}\left\|\hat{f}_{a_{i}}(t)-f_{a_{i}}(t)\right\|=a_{i} \leq \frac{1}{2} \lambda_{\min }\left(P_{i}\right)\left\|K_{s_{i}} Q_{s} P_{s} b_{i}(x) \Pi_{i}\right\|^{-1}\left\|n_{s_{i}}(t)\right\|$.

Therefore, $V_{a_{i}}(t) \leq 0$, via the application of Barbalat's lemma, we can obtain the slow subsystem (25) asymptotically stable.

After fault accommodation, the state observation error is given as:

$$
\begin{aligned}
\dot{e}_{i}(t)= & \dot{n}_{s_{i}}(t)-\dot{m}_{i}(t) \\
= & \left(A_{s}-L_{i}\right) e_{i}(t)+v_{s_{i}}\left(\left(Q_{s} K_{s_{i}}\right)^{-1} n_{s_{i}}(t)\right) \\
& -K_{s_{i}} Q_{s} P_{s} b_{i}(x) \tilde{\theta}_{i}(t) g_{i}(t)
\end{aligned}
$$

with a solution

$$
\begin{aligned}
e_{i}(t)= & e^{\left(A_{s}-L_{i}\right)\left(t-T_{a_{i}}\right)} e_{i}\left(T_{a_{i}}\right) \\
& +\int_{T_{a_{i}}}^{t} e^{\left(A_{s}-L_{i}\right)(t-\tau)}\left(v_{s_{i}}\left(\left(Q_{s} K_{s_{i}}\right)^{-1} n_{s_{i}}(\tau)\right)\right. \\
& \left.-K_{s_{i}} Q_{s} P_{s} b_{i}(x) \widetilde{\theta}_{i}(\tau) g_{i}(\tau)\right) d \tau .
\end{aligned}
$$

The residual signal given by the norm is

$$
\left\|e_{i}(t)\right\| \leq r_{i}(t)+\left\|\int_{T_{a_{i}}}^{t} e^{\left(A_{s}-L_{i}\right)(t-\tau)} K_{s_{i}} Q_{s} P_{s} b_{i}(x) \tilde{\theta}_{i}(\tau) g_{i}(\tau)\right\| d \tau .
$$

From the proof of Theorem 2, we have $\lim _{t \rightarrow \infty} \hat{\theta}_{i}(t)=\theta_{i}$, when $t \rightarrow \infty, \hat{\theta}_{i}(t)=0$. Hence, when $t \rightarrow \infty,\left\|e_{i}(t)\right\| \leq r_{i}(t)$.

Remark 2. It should be noted that the $i$ th fault accommodating controller is driven only by the $i$ th system state and the $i$ th actuator.

\section{NUMERICAL RESULTS}

In this section, the method for fault detection and accommodation are validated using computer simulations. Consider a thin, long rod in a reactor, which is fed with pure species $P$ and a zero order exothermic catalytic reaction of the form $P \rightarrow Q$ takes place on the rod [16]. Since the reaction is exothermic, a cooling medium in contact with the rod is used to 
facilitate temperature control. Under standard modeling assumptions, the mathematical model, which describes the spatiotemporal evolution of the dimensionless rod temperature is represented by the following DPS:

$$
\begin{aligned}
\frac{\partial z}{\partial t}= & \frac{\partial^{2} z}{\partial x^{2}}+\beta_{u}\left(\sum_{i=1}^{n} b_{i}(x)\left(u_{i}(t)+H\left(t-T_{a_{i}}\right) f_{a_{i}}(t)\right)-z\right) \\
& +\beta_{T} e^{-r(1+z)}-\beta_{T} e^{-r}
\end{aligned}
$$

with the initial condition and boundary condition:

$z(x, 0)=z_{0}(x), z(0, t)=z(\pi, t)=0$,

where $z$ denotes the dimensionless temperature of the reactor rod, $\beta_{u}$ denotes the dimensionless heat transfer coefficient, $\beta_{T}$ denotes a dimensionless heat of reaction, $r$ denotes a dimensionless activation energy. The process parameters are $\beta_{T}=50, \beta_{u}=2, r=4$.

The system of Eq. (28) can be written in the form of Eq. (2). Eigenvalue of $A$ can be solved analytically and solution is

$\lambda_{i}=-i^{2}, \phi_{i}(x)=\sqrt{\frac{2}{\pi}} \sin (i x), i=1,2, \cdots, \infty$,

where $\lambda_{i}$ and $\phi_{i}(x)$ denote the eigenvalues and eigenfunctions of $A$, respectively. The solution verified that the operating steady state $z(t, x)=0$ is unstable due to the exothermic nature of the reaction. For this case, we consider the first four eigenvalues to be the dominant ones, a total of $n=4$ actuators located at

$x_{i}^{a}=\frac{(i-0.137) \pi}{n+1}, i=1, \cdots, 4$.

The spatial distribution of the actuating devices is considered to be of the following form:

$b_{i}(x)=\left\{\begin{array}{l}\frac{1}{\varepsilon}, x \in\left[x_{i}^{a}-\varepsilon, x_{i}^{a}+\varepsilon\right], \\ 0, \text { otherwise, }\end{array}\right.$

where $\varepsilon=0.05$. The transformation $n_{s_{i}}(t)=K_{s_{i}} Q_{s} z_{s}(t)$ can be used to obtain the approximate slow system (9). To demonstrate how the fault detection and accommodation schemes work, we initialize the closed-loop systems (9) and filters (11) using actuators $i$ for $i=1, \cdots, 4$.

The case, where fault occurs in a single actuator is considered first. Fig. (1) shows the evolution of residual signals for the four systems with fixed and time-varying thresholds. In Fig. (1a), a fault is introduced in actuator \#1. The fault occurs at $T_{a_{i}}=1 \mathrm{~s}$. When using a time-varying threshold, the system detects the fault at $t_{a_{i}}=2.5 \mathrm{~s}$. Then, using fault accommodation, the residual signal asymptotically reaches an ideal state. When using a fixed threshold, the fault is detected at $t=4.01 \mathrm{~s}$. (The residual signal denoted by $\hat{e}_{i}(t)$ with fixed threshold). As shown in Figs. (1b)-(1d), the three residual signals do not ex- ceed their specified thresholds, indicating that no faults can be detected in actuators \#2 to \#4.

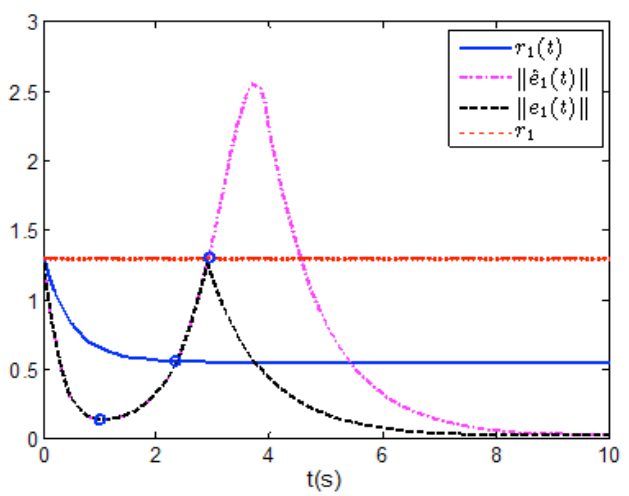

(a)

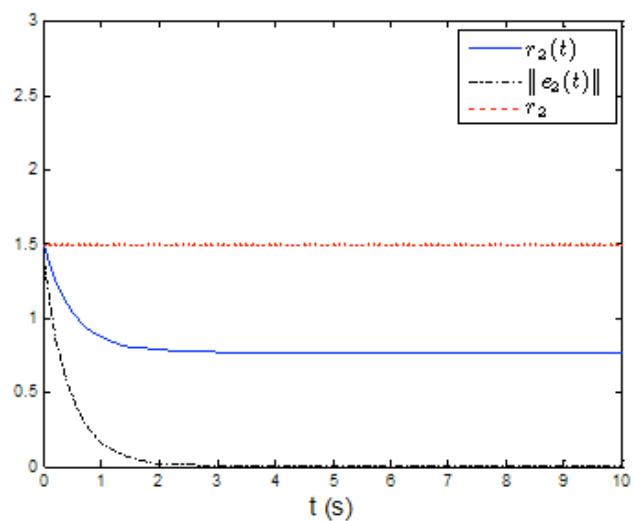

(b)

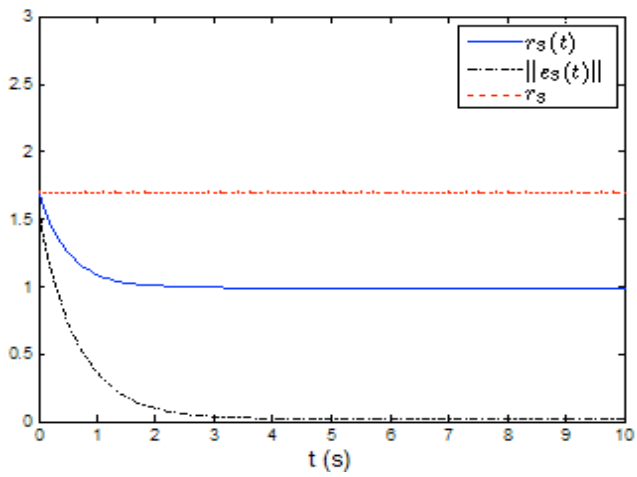

(c)

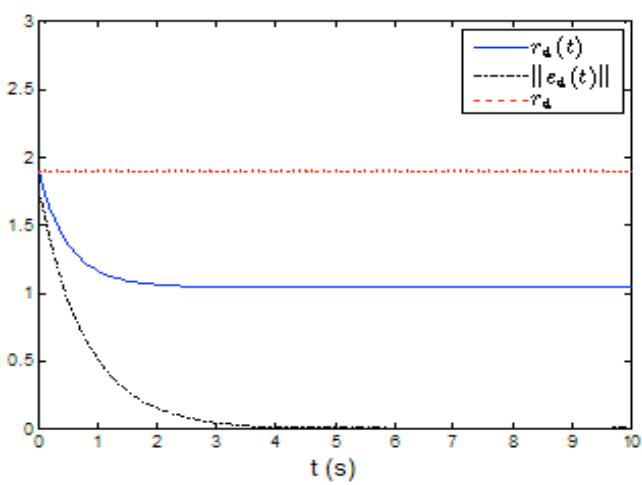

(d)

Fig. (1). Evolution of residual signals when fault in actuator \#1. 


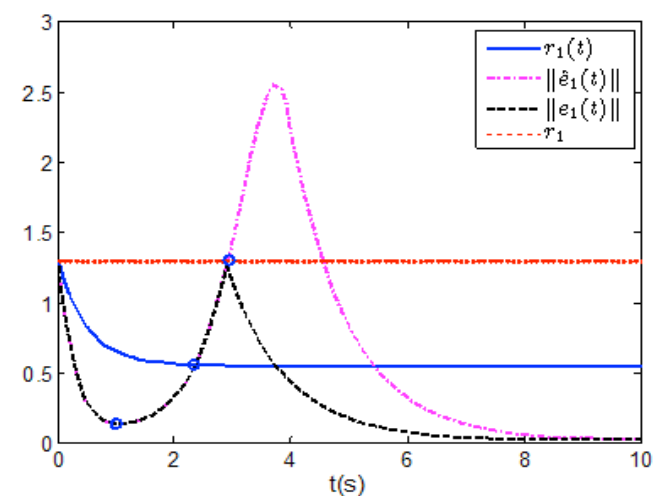

(a)

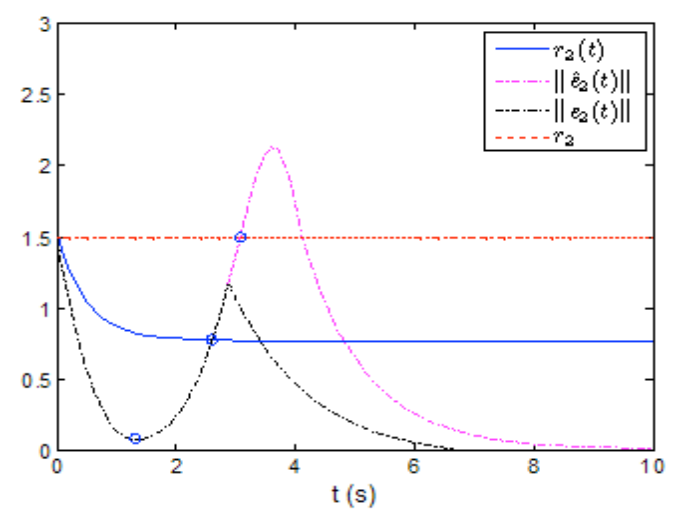

(b)

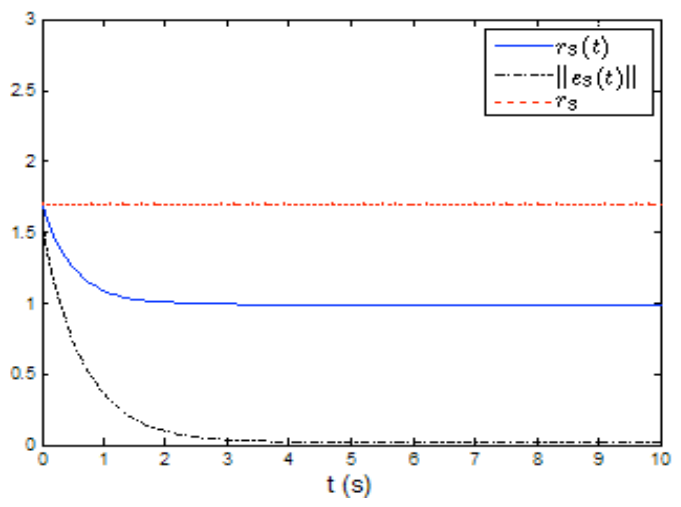

(c)

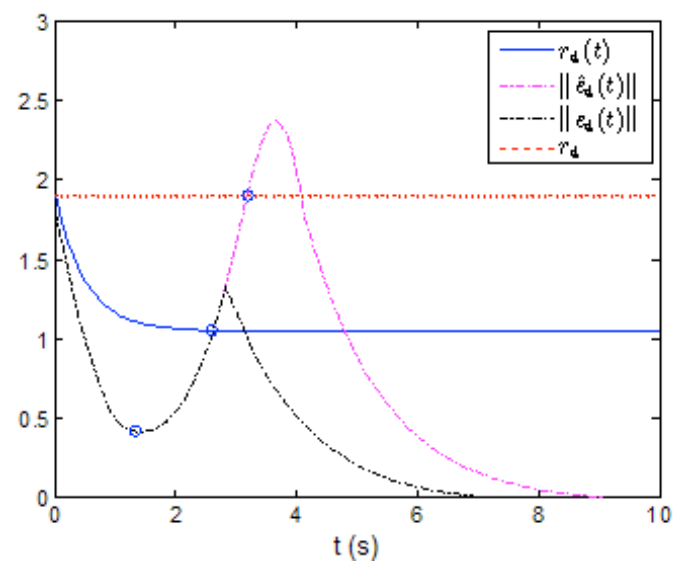

(d)
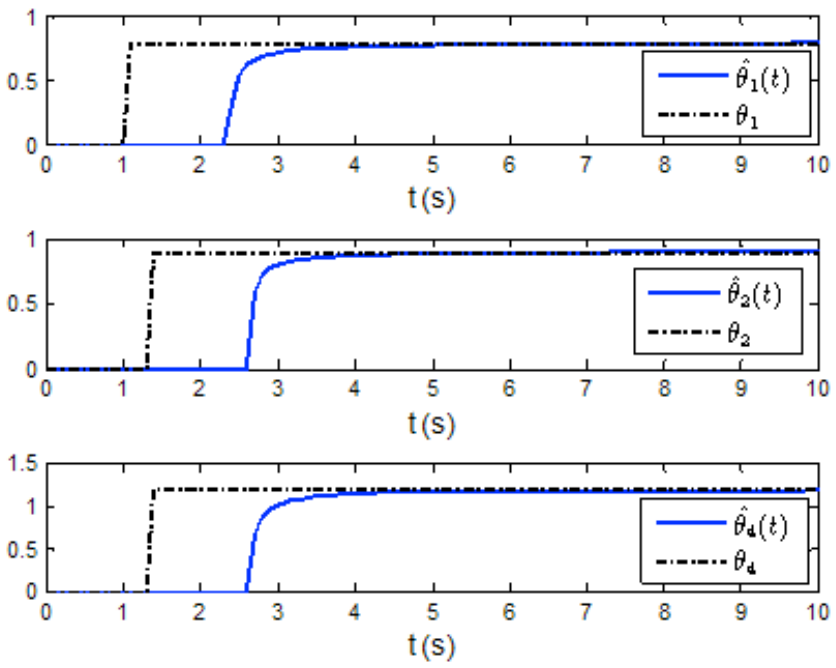

Fig. (3). Evolution of fault parameters and their adaptive estimates.

Figs. (2a), (2b) and (2d) show the results when actuator \#1, actuator \#2 and actuator \#4 experience faults. The faults were declared at $t_{a_{1}}=2.5 \mathrm{~s}, t_{a_{2}}=2.8 \mathrm{~s}$ and $t_{a_{4}}=2.82 \mathrm{~s}$. A faster convergence is observed when time-varying thresholds are used for fault detection. Fig. (2c) depict the case when no fault is introduced in actuator \#3. As expected, the faults in actuator $\# 1$, actuator \#2 and actuator \#4 do not affect the healthy actuator \#3. The evolution of the actuator fault parameters and their adaptive on-line estimates are presented in Fig. (3). It can be seen that $\hat{\theta}_{i}(t)$ converges to the desired value, $i=1,2,4$.

\section{CONCLUSION}

In this paper, the actuator fault detection and accommodation for a class of DPSs has been presented. Model reduction technique has initially been utilized to derive finitedimensional approximations that capture the dominant dynamics of the DPSs. A set of detection observers have been proposed that separately monitor each actuator fault. A fault was declared when the residual signal exceeded its time-varying threshold. Once a fault is detected, a fault diagnostic scheme using online approximation of the fault with adaptive estimate will be developed. To preserve closed-loop stability and reduce deterioration of system performance resulting from actuator fault, a control reconfiguration scheme has been implemented. The effectiveness of the proposed strategy has been successfully applied and demonstrated on a typical DPS.

\section{CONFLICT OF INTEREST}

The authors confirm that this article content has no conflicts of interest.

\section{ACKNOWLEDGEMENTS}

This paper was supported by the National Natural Science Foundation of China (61174021).

Fig. (2). Evolution of residual signals when faults in three actuators. 


\section{REFERENCES}

[1] L. Cai, X. Tian, "A new fault detection method for non-Gaussian process based on robust independent component analysis", Process Safety and Environmental Protection, vol. 92, no. 6, pp. 645-658, 2013.

[2] J. Huang, L. Yu, M. Zhang, F. Zhu, Z. Han, "Actuator fault detection and estimation for the Lur'e differential inclusion system", Applied Mathematical Modelling, vol. 38, no. 7, pp. 2090-2100, 2014.

[3] C. Hajiyev, "Generalized Rayleigh quotient based innovation covariance testing applied to sensor/actuator fault detection", Measurement, vol. 47, pp. 804-812, 2014.

[4] M. Du, J. Scott, P. Mhaskar, "Actuator and sensor fault isolation of nonlinear process systems", Chemical Engineering Science, vol. 104, pp. 294-303, 2013.

[5] X.G. Yan, "Edwards C. Robust sliding mode observer-based actuator fault detection and isolation for a class of nonlinear systems", International Journal of Systems Science, vol. 39, no. 4, pp. 349359, 2008.

[6] C, Liu, B. Jiang, " $H_{\infty}$ fault-tolerant control for time-varied actuator fault of nonlinear system”, International Journal of Systems Science, vol. 45, no. 12, pp. 2447-2477, 2014.

[7] E. Aggelogiannaki, H. Sarimveis, "Nonlinear model predictive control for distributed parameter systems using data driven artificial neural network models", Computers and Chemical Engineering, vol. 32, no. 6, pp. 1225-1237, 2008.
[8] E. Aggelogiannaki, H. Sarimveis, "Robust nonlinear $H_{\infty}$ control of hyperbolic distributed parameter systems", Control Engineering Practice, vol. 17, no. 6, pp. 723-732, 2009.

[9] N.H. El-Farra, "Integrated fault detection and fault-tolerant control architectures for distributed processes", Industrial and Engineering Chemistry Research, vol. 45, no. 25, pp. 8338-8351, 2006.

[10] N.H. EI-Farra and S. Ghantasala, "Actuator fault isolation and reconfiguration in transport-reaction processes", AIChE Journal, vol. 53, no. 6, pp. 1518-1537, 2007.

[11] M.A. Demetriou, K. Ito, R.C. Smith, "Adaptive monitoring and accommodation of nonlinear actuator faults in positive real infinite dimensional systems", IEEE Transactions on Automatic Control, vol. 52, no. 12, pp. 2332-2338, 2007.

[12] M.A. Demetriou and M.M. Polycarpou, "Incipient fault diagnosis of dynamical systems using online approximators" IEEE Transactions on Automatic Control, vol. 43, no. 11, pp. 1612-1617, 1998.

[13] A. Armaou and M.A. Demetriou, "Robust detection and accommodation of incipient component and actuator faults in nonlinear distributed processes", AIChE Journal, vol. 54, no. 10, pp. 2651-2662, 2008.

[14] P.D. Christofides and P. Daoutidis, "Finite-dimensional control of parabolic pde systems using approximate inertial manifolds", Journal of Mathematical Analysis and Applications, vol. 216, no. 2, pp 398-420, 1997.

[15] J.J.E. Slotine, W.P. Li, Applied Nonlinear Control, China Machine Press, Beijing, pp. 122-126, 2004.

[16] P.D. Christofides, Nonlinear and Robust Control of Partial Differential Equation Systems: Methods and Applications to TransportReaction Processes, Birkhäuser, Boston, pp. 153-180, 2001.

(C) Mu and Cui; Licensee Bentham Open.

This is an open access article licensed under the terms of the Creative Commons Attribution Non-Commercial License (http://creativecommons.org/licenses/by$\mathrm{nc} / 4.0 /$ ), which permits unrestricted, non-commercial use, distribution and reproduction in any medium, provided the work is properly cited. 